\title{
Effects of Stress on Lithium Transport in Amorphous Silicon Electrodes for Lithium-ion Batteries
}

\author{
Jie Pan ${ }^{\mathrm{a}}$, Qinglin Zhang, ${ }^{\mathrm{a}, \mathrm{b}}$, Juchuan Li ${ }^{\mathrm{a}, 1}$, Matthew J. Beck ${ }^{\mathrm{a}, \mathrm{c}}$, Xingcheng \\ Xiao ${ }^{\mathrm{b}}$, Yang-Tse Cheng ${ }^{\mathrm{a}, *}$ \\ ${ }^{a}$ Department of Chemical and Materials Engineering, University of Kentucky, Lexington, \\ Kentucky 40506, United States \\ ${ }^{b}$ Chemical Sciences and Materials Systems Laboratory, General Motors Global Research and \\ Development Center, Warren, Michigan 48090, United States \\ ${ }^{c}$ Center for Computational Sciences, University of Kentucky, Lexington, Kentucky 40506, \\ United States
}

\begin{abstract}
Silicon, as a promising electrode material for high energy density lithium ion batteries, experiences large strains and stresses during lithiation and delithation. The coupling effect between stress and lithium diffusion leads to a grand challenge to optimizing the design of Si electrodes with high capacity and high rate capability, particularly considering the amorphization of Si at initial cycles. In this study, we established a relationship between stress and the diffusion coefficients of Li in amorphous $\mathrm{Si}$ by ab initio molecular dynamics calculations (AIMD). The prediction from AIMD was validated by the potentiostatic intermittent titration measurements. Our results showed that two Li diffusion mechanisms can operate depending on the stress state. Specifically, the stress can increase Li diffusion either through increasing free volume under tension or by changing local structure under compression. However, within the range of stress generated during the lithiation and delithation process, diffusion coefficients are expected to vary by only one order of magnitude.
\end{abstract}

Keywords: lithium ion battery, stress, diffusion, silicon, ab initio molecular

\footnotetext{
${ }^{*}$ Corresponding author. Tel: +1 (859)323-4191; Fax: +1 (859)323-1929

Email addresses: jie.pan@uky.edu (Jie Pan), qinglinzhang@uky.edu (Qinglin Zhang), jlin@g.uky.edu (Juchuan Li ), beck@engr.uky.edu (Matthew J. Beck), xingcheng.xiao@GM.COM (Xingcheng Xiao), yang.t.cheng@uky.edu (Yang-Tse Cheng)

${ }^{1}$ Present address: Oak Ridge National Laboratory, Oak Ridge, Tennessee 37830, USA
}

Preprint submitted to Nano Energy

January 28, 2015

(C) 2015. This manuscript version is made available under the Elsevier user license http://www.elsevier.com/open-access/userlicense/1.0/ 
dynamics, potentiostatic intermittent titration technique 


\section{Introduction}

In recent years, silicon $(\mathrm{Si})$ has received much interest as a promising negative electrode material for lithium ion batteries (LIBs) because of its high specific capacity, low discharge voltage, and low cost.[1, 2, 3, 4] However, these merits come with a range of problems: large volume expansion upon cycling ( 300\%), cracking, and structural changes $[5,6]$, leading to severe capacity degradation and short cycle life. Furthermore, lithium (Li) transport in $\mathrm{Li}_{x} \mathrm{Si}$ electrodes is slow with a diffusion coefficient approximately between $10^{-10}$ and $10^{-14} \mathrm{~cm}^{2} \mathrm{~s}^{-1}[7,8,9,10]$. The performance of LIBs at high charge/discharge rates (i.e., high current densities) critically depends on the Li transport properties in electrodes. To limit the impact of these challenges, nano-structured $\mathrm{Si}$ systems (e.g., nanowires [11, 12, 13, 14, 15], nanotubes [16, 17], nanoparticles $[18,19]$, and patterned nanostructures [20]) have been proposed to meet the demands for high performance batteries. In these electrode structures, improved rate-performance is made by reducing the effective diffusion distance that $\mathrm{Li}$ must travel. These improvements are achieved at the cost of (1) severe solid electrolyte interface (SEI) formation due to large surface area and (2) relatively low energy density. In order to design Si electrodes with required performance and durability, careful engineering is needed to balance the intrinsic properties of $\mathrm{Si}$ and nanostructuring. Thus, it is necessary, in the context of charge/discharge rate, to understand the detailed mechanism of Li diffusion in Si electrodes.

One the other hand, the coupling effect of lithium diffusion and its induced stress makes the understanding of lithium transport more difficult, since stress can impact Li diffusion and vice versa. Although several publications have reported Li diffusion coefficients in $\mathrm{Si}[7,8,9,10]$, the effect of stress on diffusion behavior has not been reported. Large stresses on the order of a few GPa [21, 22] have been observed during lithiation and delithiation of $\mathrm{Si}$. In most cases, $\mathrm{Si}$ electrodes are amorphous. Unfortunately, there is no universal rule which can describe the effects of hydrostatic stress on diffusion [23] in amorphous alloys. In some amorphous systems, the hydrostatic compressive stress would increase 
the diffusion coefficient (e.g., the viscosity decreased with pressure in $\mathrm{NaAlSi}_{2} \mathrm{O}_{6}$ melts [24], and enhanced interdiffusion in amorphous $\mathrm{Si} /$ Ge multilayers [25]), while in some other amorphous systems the effects of pressure on diffusion are absent (e.g., ${ }^{57} \mathrm{Co}$ diffusion in $\mathrm{Co}_{81} \mathrm{Zr}_{19}$ [26]). In addition, it was found that a compressive stress could decrease the diffusion rates in some metallic glasses (e.g., ${ }^{95} \mathrm{Zr}$ diffusion in $\left.\mathrm{Co}_{92} \mathrm{Zr}_{8}[27]\right)$. The fact that $\mathrm{Si}$ experiences both compressive and tensile stresses during electrode cycling raised additional complexities. Therefore, it is necessary to study the detailed kinetics and structural changes under hydrostatic tension and compression in order to understand Li transport in amorphous $\mathrm{Si}$.

In this paper, we present a study integrating both calculations and experiments to investigate the effect of stress on Li diffusion in Si electrodes. Explicit ab initio molecular dynamics (AIMD) simulations based on density functional theory (DFT) were performed to study the changes in structure, diffusion rates, and transport mechanisms in stressed LiSi systems. Our calculations found that Li diffusion coefficients vary within one order of magnitude under the stresses encountered in $\mathrm{Si}$ electrodes, in agreement with experimental observations of $\mathrm{Li}$ diffusion coefficients in lithiated Si. The results of this work provide a better understanding at the atomic level of $\mathrm{Li}$ diffusion behavior in amorphous $\mathrm{Si}$, which can also be helpful to the understanding of diffusion in other similar inorganic amorphous systems.

\section{Methodology}

\subsection{Computational Details}

$A b$ initio Molecular Dynamics simulations, which provide fundamental information about diffusion at the atomic scale, were performed using the Vienna Ab initio Simulation Package (VASP) [28, 29]. The exchange and correlation function was approximated at the level of the generalized gradient approximation (GGA) in the Perdew-Burke-Ernzerhof (PBE) flavor [30]. In addition, the ionic cores were modeled with projector-augmented-wave (PAW) potentials 
[31]. The Brillouin zone was sampled at the $\Gamma$ point, which represented about $3.3 \times 10^{-4} \AA^{-3}$ k-points for the selected box size. The energy cutoff for the planewave expansion of the electronic eigenfunctions was $350 \mathrm{eV}$. These parameters were selected based on convergence tests of calculated total energy, and were comparable with the parameters used in previous calculations [32].

In the present calculations, we focus on amorphous LiSi (atomic ratio 1:1) composition as an example of a range of amorphous $\mathrm{Li}_{x} \mathrm{Si}$ materials relevant to Si based LIB electrodes. Amorphous LiSi alloy structures were prepared by AIMD within VASP $[28,29]$. The systems were first created as crystals, typically containing 192 atoms (96 atoms for each species). These structures were then heated up to a temperature above the melting temperature [33] (e.g., $1700 \mathrm{~K}$ ). At this temperature, systems were equilibrated for $8000 \mathrm{MD}$ steps with a $0.5 \mathrm{fs}$ time step. To obtain amorphous structures relevant at lower temperatures, the systems were then quenched to a temperature of interest to perform structural studies and diffusion calculations (e.g., $1200 \mathrm{~K}$ and $900 \mathrm{~K}$ ) with a rapid cooling rate: $200 \mathrm{~K}$ per 2000 time steps (1000 fs), similar to the cooling rates used in previous calculations $[34,32]$. To prepare systems with different stresses at a specific temperature, we performed AIMD simulations at various volumes by adjusting the simulation box size from above to below the equilibrium volume of the stress-free state. A total of 2000 time steps ( $0.5 \mathrm{fs} / \mathrm{step}$ ) was used to equilibrate the volume controlled system. The potential energy of each system converged to a stable value (the differences in potential energy were within $10^{-4}$ $\mathrm{eV} /$ atom) after 2000 time steps, signalling the end of the equilibration phase of the AIMD simulations at a given volume. After that, the potential energy of another 2000 time steps were averaged and the energy values of different volumes were fitted to a third order polynomial with respect to the box volume $V$. (See Supporting Information for details.)

The hydrostatic stress in the simulation box is determined as

$$
\sigma_{T, N}=\left.\frac{\partial\langle E(V, T, N)\rangle}{\partial V}\right|_{T, N},
$$

where $\langle E(V, T, N)\rangle$ is the the equilibrated potential energy represented by the 
third order polynomial which is a function of the volume, $V$, of the simulation boxes, the temperature, $T$, and the number of atoms, $N$. The equilibrium volume (stress-free volume) at different temperatures correspond to the volume $V_{\text {equi }}$ at which

$$
\frac{\partial\langle E\rangle}{\partial V}=\frac{1}{3 a_{0}^{2}} \frac{\partial\langle E\rangle}{\partial a_{0}}=0,
$$

where $a_{0}$ is the supercell lattice constant. The equilibrated simulation box volumes corresponding to each stress state of interest (e.g., 2, 1, -1, and -2 GPa) were calculated from Equation 1 and were used to construct amorphous LiSi (aLiSi) boxes at various stress states. These stressed boxes were equilibrated for 1000 time steps prior to characterization.(See Supporting Information for details.)

\subsection{Experimental Details}

Diffusion coefficients of $\mathrm{Li}$ in amorphous $\mathrm{Si}$ were measured using a potentiostatic intermittent titration technique (PITT). The working electrodes were 100 $\mathrm{nm}$ thick Si films on $\mathrm{Cu}$ foils fabricated by E-beam evaporation, and the counter electrodes were pure Li metals. The electrolyte was $1 \mathrm{M} \mathrm{LiPF}_{6}$ salt in 1:1 ratio of ethylene carbonate/dimethyl carbonate (EC/DMC). PITT experiments were conducted using a Bio-Logic potentiostat (VMP3) at room temperature. Potential steps of $5 \mathrm{mV}$ were applied until the current was bellow $1 \mathrm{~mA} \mathrm{~g}^{-1}$, equivalent to a rate of $\mathrm{C} / 4200$. Diffusion coefficients were obtained using the intercept of long times $(\tau \gg 1)[35]$ and the interfacial kinetics were considered to be much faster compared with diffusion $[7,36]$.

The stresses in the thin film was measured by the in-situ Multi-beam Optical Stress Sensor (MOSS) stress measurement method[37, 38, 39]. Thin film amorphous silicon samples of $100 \mathrm{~nm}$ were deposited by e-beam evaporation system on quartz-titanium substrates. Before depositing Si, a $200 \mathrm{~nm}$ thick titanium layer was deposited on the quartz as the current collector. In addition, the titanium improves the adhesion between amorphous silicon and the substrate. The custom-made cells were subjected to galvanostatic cycles (constant currents) against lithium metal between $1.5 \mathrm{~V}$ and $0.05 \mathrm{~V}$ with a rate 
of $\mathrm{C} / 10$. The back surface of the quartz wafer was visible through a quartz window on top of the sealed cell, which allowed the laser of the MOSS system to go through to monitor the bending of thin film electrodes. By monitoring the bending with MOSS, the curvature of substrate was obtained. Within the stress level, the quartz substrate deformed elastically. The bi-axil stress in the film was calculated from the curvature $\kappa$ by Stoney equation [40]

$$
\sigma_{f}=\frac{E_{s} h_{s}^{2} \kappa}{6 h_{f}\left(1-\nu_{s}\right)},
$$

where $E_{s}$ is the Young's modulus of substrate, $h_{s}$ the substrate thickness, $h_{f}$ is the film thickness, and $v_{s}$ is the Poisson's ratio of substrate.

\section{Results and Discussions}

The partial radial distribution function (RDF), namely the second order correlation function $g(\mathbf{r})$, describing the probability to find an atom in a shell at the distance $r$ from a reference atom, provides a measure of how atoms are arranged in a material. In amorphous materials, it characterizes the short range order and allows structural comparisons among different systems. Partial RDFs were calculated by invoking the effective isotropy of amorphous structures as

$$
g_{\beta-\alpha}(r)=\frac{1}{\langle\rho\rangle} \frac{\mathrm{d} n_{\alpha}(r, r+\mathrm{d} r)}{\mathrm{d} v(r+d r)} \simeq \frac{1}{\langle\rho\rangle} \frac{\mathrm{d} n_{\alpha}(r, r+\mathrm{d} r)}{4 \pi r^{2} d r},
$$

where $\langle\rho\rangle$ is the averaged particle density, $\mathrm{d} n_{\alpha}(r, r+\mathrm{d} r)$ is the number of particles of type $\alpha$ in the shell $(r, r+\mathrm{d} r)$ at distance $\mathrm{r}$ of a reference atom $\beta$, $\mathrm{d} v(r+d r)$ is the volume of the shell. Figure 1 shows the calculated partial RDFs, $g_{L i-L i}(r), g_{L i-S i}(r)$ and $g_{S i-S i}(r)$ for stress-free LiSi at $1200 \mathrm{~K}$. All partial RDFs were averaged over 5000 time steps in the MD trajectory. Figure 1 shows that $\mathrm{Si}$ is the nearest neighbor to both $\mathrm{Li}$ and $\mathrm{Si}$. The $\mathrm{Si}-\mathrm{Si}$ neighbor distance is $2.4 \AA$ and it is slightly larger than the value of $2.3 \AA$ in the crystalline $\mathrm{Si}$ [41]. The nearest neighbors of $\mathrm{Li}$ are $\mathrm{Si}$ (around $2.6 \AA$ ) and the first peak of $\mathrm{Li}-\mathrm{Li}$ pair is located at $r=2.7 \AA$. That $\mathrm{Si}$ is nearest to both $\mathrm{Li}$ and $\mathrm{Si}$ is because $\mathrm{Li}$ atoms repel each other, and $\mathrm{Si}-\mathrm{Si}$ covalent bonds are still strong in 
amorphous Li-Si systems (Figure S4 in the Supporting Information). Grid-based Bader Charge analysis $[42,43,44]$ shows that the electronic state of $\mathrm{Si}$ varies from $[N e] 3 s^{2} 3 p^{2}$ in crystalline Si to $[N e] 3 s^{2} 3 p^{3}$ in the amorphous LiSi (a-LiSi), and Li atoms are positively charged. This factor explains the slight increment in the length of $\mathrm{Si}-\mathrm{Si}$ bonds [32] and the preference by $\mathrm{Li}$ atoms to have $\mathrm{Si}$ as their nearest neighbors. Comparing with previous simulation data [32], the first and second peaks in all of our $g(r)$ agree with those in the latest time step (15 ps). Furthermore, the second $g_{S i-S i}(r)$ peak in our simulation is at 4.1 to 4.3 $\AA$ and it is supported by the experimental partial RDF data in the second cycle [45]. In addition, the bulk modulus of our simulated LiSi amorphous structure is consistent with previous experimental and computational results (Table S2 in Supporting Information). Thus, our simulated structure is consistent with previous computational and experimental studies. In addition, comparing partial RDFs at 900 and $1200 \mathrm{~K}$ shows that the locations of the first peaks in the partial RDFs each shift to lower $r$ values with the lower temperature but only by $1 \%$. The order of the nearest neighbor peaks remains the same (from small to large separation: $\mathrm{Si}-\mathrm{Si}, \mathrm{Si}-\mathrm{Li}, \mathrm{Li}-\mathrm{Li}$ ).

The diffusion coefficient can be estimated from the Mean Squared Displacement (MSD) of each atom type during a finte temperature MD [46]

$$
\left\langle[\Delta R(t)]^{2}\right\rangle=\frac{1}{N} \sum_{i=1}^{N}\left|\vec{R}_{i}(t)-\vec{R}_{i}(0)\right|^{2},
$$

where $\left\langle\Delta R(t)^{2}\right\rangle$ is the MSD and $\overrightarrow{R_{i}}(t)$ are the atomic positions. The diffusion coefficients are then calculated using the Stokes-Einstein Relationship [32, 46]

$$
D_{L i}=\lim _{t \rightarrow \infty} \frac{\left\langle\left[\Delta R_{L i}(t)\right]^{2}\right\rangle}{6 t} .
$$

In this study, the diffusion coefficients of $\mathrm{Li}$ at room temperature in a-LiSi with different stress states were extrapolated from AIMD calculations at elevated temperatures (Figure S6 in Supporting Information) according to the Arrhenius Law

$$
D_{L i}(T)=D_{0} \exp \left(\frac{-\Delta G}{k_{B} T}\right)
$$


where $\Delta G$ is the effective diffusion barrier (values of $\Delta G$ are listed in Table S3 in Supporting Information), $k_{B}$ is Boltzmann constant and $T$ is temperature. The extrapolated diffusion coefficients at $300 \mathrm{~K}$ (Figure 2(a)) shows that increasing the tensile stress leads to faster Li diffusion in a-LiSi. Compression, on the other hand, results in a relatively small change in diffusivity. At $300 \mathrm{~K}$, the diffusion rate increases at -2 GPa compared with that at $-1 \mathrm{GPa}$ (compressive stress). In latter discussions, we will show that this phenomenon can be attributed to a local structural change that destablizes the $\mathrm{Li}$ in the cage formed by surrounding $\mathrm{Li}$ and $\mathrm{Si}$ atoms.

Experiments have shown that the stress during lithiation and delithiation of $\mathrm{Si}$ is several GPas [21, 22]. Therefore, based on the above simulation results, the diffusion coefficients during lithiation and delithiation are expected to be similar, with diffusivity during delithiation (under tension) trending somewhat higher than that during lithiation (under compression). This expectation was verified using the the Potentiostatic Intermittent Titration Technique (PITT) measurements, which measured diffusivity at a constant Li concentration. To minimize other effects on Li diffusion, a thin film structure is selected in the PITT measurements. Thin film structure is chosen for this study, because 1) it takes shorter time to reach uniform composition; 2) cracking can be minimized [47]; 3) the complications from binders and conducting additives are avoided. Figure 3(a) compares the diffusion coefficients at the same state-of-charge in lithiation and delithiation. The results of in-situ stress measurements in the $\mathrm{Si}$ electrode accompanied by the potential profile is shown in Figure 3(b)-(c). The experimental results indicate that within the composition range investigated in this work, i.e., $x$ between 0 and 2.7 in $\mathrm{Li}_{x} \mathrm{Si}$, the diffusion coefficients are within the same order of magnitude. The diffusion coefficients for Li under compression stress is slightly lower than those under tensile stress with the stress difference around $3 \mathrm{GPa}$. Thus, the variation of calculated diffusivity values at different stress states agree quantitatively with experimental results.

To better understand the effect of stress on diffusivity in a-LiSi, we examine the detailed structural changes induced by stress. We did this study from the 
atomic trajectories simulated at $900 \mathrm{~K}$ for the reasons that: 1) $900 \mathrm{~K}$ is below the melting temperature according to the Li-Si phase diagram [33]; 2) The trend of diffusion coefficients in response to the applied stress is the same as that at $300 \mathrm{~K}$ (Figure 2(b)). To study the structural change, we first considered stressinduced changes in the partial RDFs. If there were no changes in the short-range atomic ordering, we would expect the first peak of partial RDFs for each atomic pair to shift uniformly with $a_{0}$. However, in LiSi the first peaks of Li-Li, Si-Li, and Si-Si RDFs shift asymmetrically in response to stress. Figure 4(a) shows the volumetric changes as a function of stress. By invoking the effective isotropy of amorphous materials under hydrostatic stress, the change in one dimension would be $+2 \%$ under +2 GPa tensile stress and $-1.6 \%$ under -2 GPa compressive stress compared with the stress free state. Figure $4(\mathrm{~b})$ to $4(\mathrm{~d})$ show the partial RDFs under different stress states. The covalent Si-Si bond is found to be the most rigid bond, with the location of the first Si-Si neighbor peak insensitive to stress (Figure S7 in Supporting Information). However, the Li-Li first neighbor peak shifts $\pm 3 \%$ under respective \pm 2 GPa stress. In addition, the Si-Li first neighbor peaks shift $\pm 1.5 \%$ under \pm 2 GPa stress, respectively. These different stress-induced changes in nearest neighbor spacings represent a change in the local environment, thus influencing the diffusion rates by altering the effective activation barrier. The different strength of pair interactions in a-LiSi (from strong to weak: $\mathrm{Si}-\mathrm{Si}, \mathrm{Si}-\mathrm{Li}$, and $\mathrm{Li}-\mathrm{Li}$ ) is the reason causing the change of the local environment with stress. In addition, we note that this observation provides an atomic level understanding of the elastic softening of LiSi relative to Si $[34,48,49,50]$.

The coordination number $(\mathrm{CN})$, describing the number of atoms located within a cut-off length (usually defined as a bond length) in amorphous materials, provides a quantitative measure of the change in a local atomic environment. Previous studies in other materials systems have shown that the $\mathrm{CN}$ can vary strongly due to an applied stress, causing, in some cases, changes in diffusivity $[24,25,51]$. To calculate CNs, a physically meaningful cut-off length must be defined for each atomic pair. To account for fluctuations in the amorphous LiSi 
system compared with crystalline LiSi, we choose cut-off lengths corresponding to $110 \%$ of the equivalent bond length in crystalline LiSi [52]: $2.65 \AA$ for $\mathrm{Si}-\mathrm{Si}$, $3.0 \AA$ for $\mathrm{Li}-\mathrm{Li}$, and $3.05 \AA$ for Si-Li. The values of cut-off length are comparable with previous calculations in a-LiSi system [53]. The average CN for $\mathrm{Si}-\mathrm{Si}$ (Figure $5(\mathrm{a})$ ) is $\sim 2.5$ and varies between 4.5 and 5.2 for $\mathrm{Si}-\mathrm{Li}$ in agreement with a previous study [53] on biaxially stressed amorphous LiSi. However, we note that changes in CNs as a function of hydrostatic stress are nonuniform for different atomic pairs (Figure $5(\mathrm{~b})$ ). The most significant change is in the $\mathrm{Li}-\mathrm{Li}$ pair, where the $\mathrm{CN}$ increases by 1.0 as the stress changes from $-2 \mathrm{GPa}$ to $2 \mathrm{GPa}$. In addition, from the MD trajectories, the diffusion process of $\mathrm{Li}$ at low temperatures can be described as discrete movements: vibrating in a cage formed by its surrounding atoms then, after a while, it jumps from one cage to another (cage depicts the structure formed by surrounding $\mathrm{Li}$ and $\mathrm{Si}$ atoms, within which the target diffusive Li vibrates). As a result, Li atoms are adjacent to significantly more $\mathrm{Li}$ atoms under compression. Since $\mathrm{Li}$ is positively charged in $\mathrm{LiSi}$, this local structural change represents an destabilization of $\mathrm{Li}$ within cages which can facilitate the diffusion of $\mathrm{Li}$.

Considering the change of diffusivity under tension, we note that the pressure dependence of diffusion can be characterized by an "activation volume" $\Delta V$ $[23,25]$,

$$
\Delta V=\left[-\frac{1}{R T} \frac{\partial \ln \left(D / D_{0}\right)}{\partial P}\right]_{T},
$$

where $P$ is pressure (compressive stress is positive). For equilibrium systems, $\Delta V$ consists of two components [25]: $\Delta V_{d}$, the volumetric change due to the formation of a defect and $\Delta V_{m}$, the variation of volume when the diffusing atom moves from a normal site to a saddle point,

$$
\Delta V=\Delta V_{d}+\Delta V_{m}
$$

From this perspective, stress influences diffusion by two related mechanisms. First, the tensile stress can increase diffusivity by increasing the available volume for defect formation, $\Delta V_{d}[23,54]$. Second, for negative values of $\Delta V_{m}$, a compressive stress can increase diffusion. A negative $\Delta V_{m}$ has been recognized 
in other $\mathrm{Si}$ or Ge containing systems $[25,24,51]$. Our results suggest that the activation volume for Li migration in LiSi is negative. As a result, tensile stress can increase diffusivity on the condition that $\left|\Delta V_{d}\right|>\left|\Delta V_{m}\right|$, and compression can increase diffusivity on the condition that $\left|\Delta V_{m}\right|>\left|\Delta V_{d}\right|$. The possible reason for $\Delta V_{m}<0$ is the open covalent network structure in the system [25]. According to partial RDFs (Figure 4(c)), the nearest neighbors of $\mathrm{Li}$ are $\mathrm{Si}$ atoms under all stress states in the LiSi system. As a result, Li diffusion at low temperature in LiSi requires Li jumping through cages formed by the surrounding Si atoms. Since the rigid nature of the Si-Si bond, Li diffusion must be accompanied by Si-Si bond angle distortion to allow diffusive motion. This bond angle fluctuation can cause $\Delta V_{m}<0$ as found in a previous study [25].

\section{Conclusions}

We combined computational and experimental methods to study stress effects on Li diffusion in Si electrodes. Based on atomic scale AIMD simulations, we found that the Li diffusion coefficient is not a monotonic function of stress ranging from tensile to compression. This occurs because the local atomic structure around Li changes under stress, causing changes in bonding between the diffusing $\mathrm{Li}$ and its neighboring atoms. Thus, the diffusion barrier can decrease with increasing compressive stress. As a result of the competition of free volume and local environment, the diffusion coefficients are expected to vary by one order of magnitude during lithiation and delithiation. This provides useful information for further optimizing the design of Si based nanostructure in order to control the Li diffusion and its induced stress/strain.

\section{Acknowledgements}

We gratefully acknowledge the support from the Center for Computational

Sciences at University of Kentucky, National Science Foundation (CMMI \#1000726), National Science Foundation Award No.1355438 (Powering the Kentucky Bioe- 
conomy for a Sustainable Future), Department of Energy and the Assistant Secretary for Energy Efficiency and Renewable Energy (Office of Vehicle Technologies of the U.S. Department of Energy under Contract No. DE-AC0205CH11231, Subcontract No. 7056410) under the Batteries for Advanced Transportation Technologies (BATT) Program. Q.Z. is grateful to GM Global Research \& Development for providing a summer internship.

\section{References}

[1] R. Mukherjee, R. Krishnan, T. M. Lu, N. Koratkar, Nano Energy 1 (4) (2012) 518-533.

[2] M. T. McDowell, S. W. Lee, W. D. Nix, Y. Cui, Adv. Mater. 25 (2013) 4966-85.

[3] X. H. Wang, L. N. Sun, R. A. Susantyoko, Y. Fan, Q. Zhang, Nano Energy 8 (2014) 71-77.

[4] L. B. Hu, N. Liu, M. Eskilsson, G. Y. Zheng, J. McDonough, L. Wagberg, Y. Cui, Nano Energy 2 (1) (2013) 138-145.

[5] L. Y. Beaulieu, T. D. Hatchard, A. Bonakdarpour, M. D. Fleischauer, J. R. Dahn, J. Electrochem. Soc. 150 (2003) A1457-A1464.

[6] B. A. Boukamp, G. C. Lesh, R. A. Huggins, J. Electrochem. Soc. 128 (1981) $725-729$.

[7] J. C. Li, X. C. Xiao, F. Q. Yang, M. W. Verbrugge, Y. T. Cheng, J. Phys. Chem. C 116 (2012) 1472-1478.

[8] N. Ding, J. Xu, Y. X. Yao, G. Wegner, X. Fang, C. H. Chen, I. Lieberwirth, Solid State Ionics 180 (2009) 222-225.

[9] J. Xie, N. Imanishi, T. Zhang, A. Hirano, Y. Takeda, O. Yamamoto, Mater. Chem. Phys. 120 (2010) 421-425. 
[10] N. Balke, S. Jesse, Y. Kim, L. Adamczyk, A. Tselev, I. N. Ivanov, N. J. Dudney, S. V. Kalinin, Nano Lett. 10 (2010) 3420-3425.

[11] M. T. McDowell, S. W. Lee, C. M. Wang, Y. Cui, Nano Energy 1 (3) (2012) 401-410.

[12] T.-L. Chan, J. R. Chelikowsky, Nano Lett. 10 (2010) 821-825.

[13] C. K. Chan, H. L. Peng, G. Liu, K. McIlwrath, X. F. Zhang, R. A. Huggins, Y. Cui, Nat. Nanotechnol. 3 (2008) 31-35.

[14] C. K. Chan, R. N. Patel, M. J. O'Connell, B. A. Korgel, Y. Cui, Acs Nano 4 (2010) 1443-1450.

[15] K. Kang, H. S. Lee, D. W. Han, G. S. Kim, D. Lee, G. Lee, Y. M. Kang, M. H. Jo, Appl. Phys. Lett. 96 (2010) 053110.

[16] T. Song, J. L. Xia, J. H. Lee, D. H. Lee, M. S. Kwon, J. M. Choi, J. Wu, S. K. Doo, H. Chang, W. Il Park, D. S. Zang, H. Kim, Y. G. Huang, K. C. Hwang, J. A. Rogers, U. Paik, Nano Lett. 10 (2010) 1710-1716.

[17] M. H. Park, M. G. Kim, J. Joo, K. Kim, J. Kim, S. Ahn, Y. Cui, J. Cho, Nano Lett. 9 (2009) 3844-3847.

[18] H. Ma, F. Y. Cheng, J. Chen, J. Z. Zhao, C. S. Li, Z. L. Tao, J. Liang, Adv. Mater. 19 (2007) 4067-4070.

[19] Y. Z. Fu, A. Manthiram, Nano Energy 2 (6) (2013) 1107-1112.

[20] S. H. Nam, K. S. Kim, H. S. Shim, S. H. Lee, G. Y. Jung, W. B. Kim, Nano Lett. 11 (2011) 3656-3662.

[21] V. A. Sethuraman, V. Srinivasan, A. F. Bower, P. R. Guduru, J. Electrochem. Soc. 157 (2010) A1253-A1261.

[22] S. K. Soni, B. W. Sheldon, X. C. Xiao, M. W. Verbrugge, D. Ahn, H. Haftbaradaran, H. J. Gao, J. Electrochem. Soc. 159 (2012) A38-A43. 
[23] F. Faupel, W. Frank, M. P. Macht, H. Mehrer, N. V, K. Ratzke, H. R. Schober, S. K. Sharma, H. Teichler, Rev. Mod. Phys. 75 (2003) 237-280.

[24] I. Kushiro, J. Geophys. Res. 81 (1976) 6347-6350.

[25] S. D. Theiss, F. Spaepen, M. J. Aziz, Appl. Phys. Lett. 68 (1996) 12261228.

[26] P. Klugkist, K. Ratzke, S. Rehders, P. Troche, F. Faupel, Phys. Rev. Lett. 80 (1998) 3288-3291.

[27] P. Klugkist, K. Ratzke, F. Faupel, Phys. Rev. Lett. 81 (1998) 614-617.

[28] G. Kresse, J. Furthmuller, Phys. Rev. B 54 (1996) 11169-11186.

[29] G. Kresse, J. Furthmuller, Comp. Mater. Sci. 6 (1996) 15-50.

[30] J. P. Perdew, K. Burke, M. Ernzerhof, Phys. Rev. Lett. 77 (1996) 38653868.

[31] G. Kresse, D. Joubert, Phys. Rev. B 59 (1999) 1758-1775.

[32] P. Johari, Y. Qi, V. B. Shenoy, Nano Lett. 11 (2011) 5494-5500.

[33] H. Okamoto, J. Phase Equilib. Diff. 30 (2009) 118-119.

[34] V. B. Shenoy, P. Johari, Y. Qi, J. Power Sources 195 (2010) 6825-6830.

[35] C. J. Wen, R. A. Huggins, J. Solid State Chem. 35 (1980) 376-384.

[36] J. Li, X. Xiao, Y.-T. Cheng, M. W. Verbrugge, J. Phys. Chem. Lett. 0 (2013) 3387-3391.

[37] V. A. Sethuraman, M. J. Chon, M. Shimshak, V. Srinivasan, P. R. Guduru, J. Power Sources 195 (2010) 5062-5066.

[38] B. W. Sheldon, S. K. Soni, X. C. Xiao, A. Tokranov, Scripta Materialia 64 (4) (2011) 307-310. 
[39] S. K. Soni, B. W. Sheldon, X. C. Xiao, A. F. Bower, M. W. Verbrugge, Journal of the Electrochemical Society 159 (9) (2012) A1520-A1527.

[40] L. Freund, S. Suresh, Thin film materials: stress, defect formation and surface evolution, Cambridge University Press, 2004.

[41] M. K. Y. Chan, C. Wolverton, J. P. Greeley, J. Am. Chem. Soc. 134 (2012) 14362-14374.

[42] W. Tang, E. Sanville, G. Henkelman, J. Phys.: Condens. Mat. 21 (2009) 084294.

[43] E. Sanville, S. D. Kenny, R. Smith, G. Henkelman, J. Comput. Chem. 28 (2007) 899-908.

[44] G. Henkelman, A. Arnaldsson, H. Jonsson 36 (2006) 354-360.

[45] B. Key, M. Morcrette, J. M. Tarascon, C. P. Grey, J. Am. Chem. Soc. 133 (2011) 503-512.

[46] J. R. Chelikowsky, J. J. Derby, V. V. Godlevsky, M. Jain, J. Y. Raty, J. Phys.: Condens. Mat. 13 (2001) R817-R854.

[47] J. C. Li, A. K. Dozier, Y. C. Li, F. Q. Yang, Y. T. Cheng, Journal of the Electrochemical Society 158 (2011) A689-A694.

[48] Z. D. Zeng, N. Liu, Q. S. Zeng, Y. Ding, S. X. Qu, Y. Cui, W. L. Mao, J. Power Sources 242 (2013) 732-735.

[49] V. L. Chevrier, J. W. Zwanziger, J. R. Dahn, Can. J. Phys. 87 (2009) 625-632.

[50] H. Kim, C.-Y. Chou, J. G. Ekerdt, G. S. Hwang, J. Phys. Chem. C 115 (2011) 2514-2521.

[51] H. S. Waff, Geophys. Res. Lett. 2 (1975) 193-196.

[52] V. L. Chevrier, J. W. Zwanziger, J. R. Dahn, J. Alloy. Compd. 496 (2010) $25-36$. 
[53] K. J. Zhao, G. A. Tritsaris, M. Pharr, W. L. Wang, O. Okeke, Z. G. Suo, J. J. Vlassak, E. Kaxiras, Nano Lett. 12 (2012) 4397-4403.

[54] P. Shewmon, Diffusion in solids, McGraw-Hill series in materials science and engineering, McGraw-Hill, 1963. 


\section{Figure Captions}

Figure 1: The Partial Radial Distribution Function of Si-Si, Li-Si, and Li-Li pairs in a-LiSi at $1200 \mathrm{~K}$.

Figure 2: (a) The relative diffusion coefficients $\left(D_{L i} / D_{L i}(\sigma=0 G P a)\right)$ as a function of stress at $300 \mathrm{~K}$. Insert: The extrapolation of diffusion coefficients at $300 \mathrm{~K}$ from high temperature calculations; (b) The relative diffusion coefficients $\left(D_{L i} / D_{L i}(\sigma=0 G P a)\right)$ as a function of stress at $900 \mathrm{~K}$. Insert: The MSD of $\mathrm{Li}$ in a-LiSi at $900 \mathrm{~K}$, the lowest temperature in our simulation, with different hydrostatic (positive value means tensile stress and negative value means compressive stress) stress states.

Figure 3: (a) Diffusion coefficients during lithiation and delithiation at different Li concentrations; (b) nominal stress calculated by Stony equation vs. $x$ in $\mathrm{Li}_{x} \mathrm{Si}$ under charge/discharge rate of $\mathrm{C} / 10$. (c) cell voltage vs. $x$ in $\mathrm{Li}_{x} \mathrm{Si}$ during stress measurement.

Figure 4: (a) The volume of the simulation boxes under hydrostatic stress at 900 K. (b)-(d) The change of the first peak in partial RDFs under different hydrostatic pressure states at $900 \mathrm{~K}$.

Figure 5: (a) The coordination number as a function of stress at $900 \mathrm{~K}$; (b) $\triangle \mathrm{CN}$ with reference of $\mathrm{CN}$ at stress free state. 


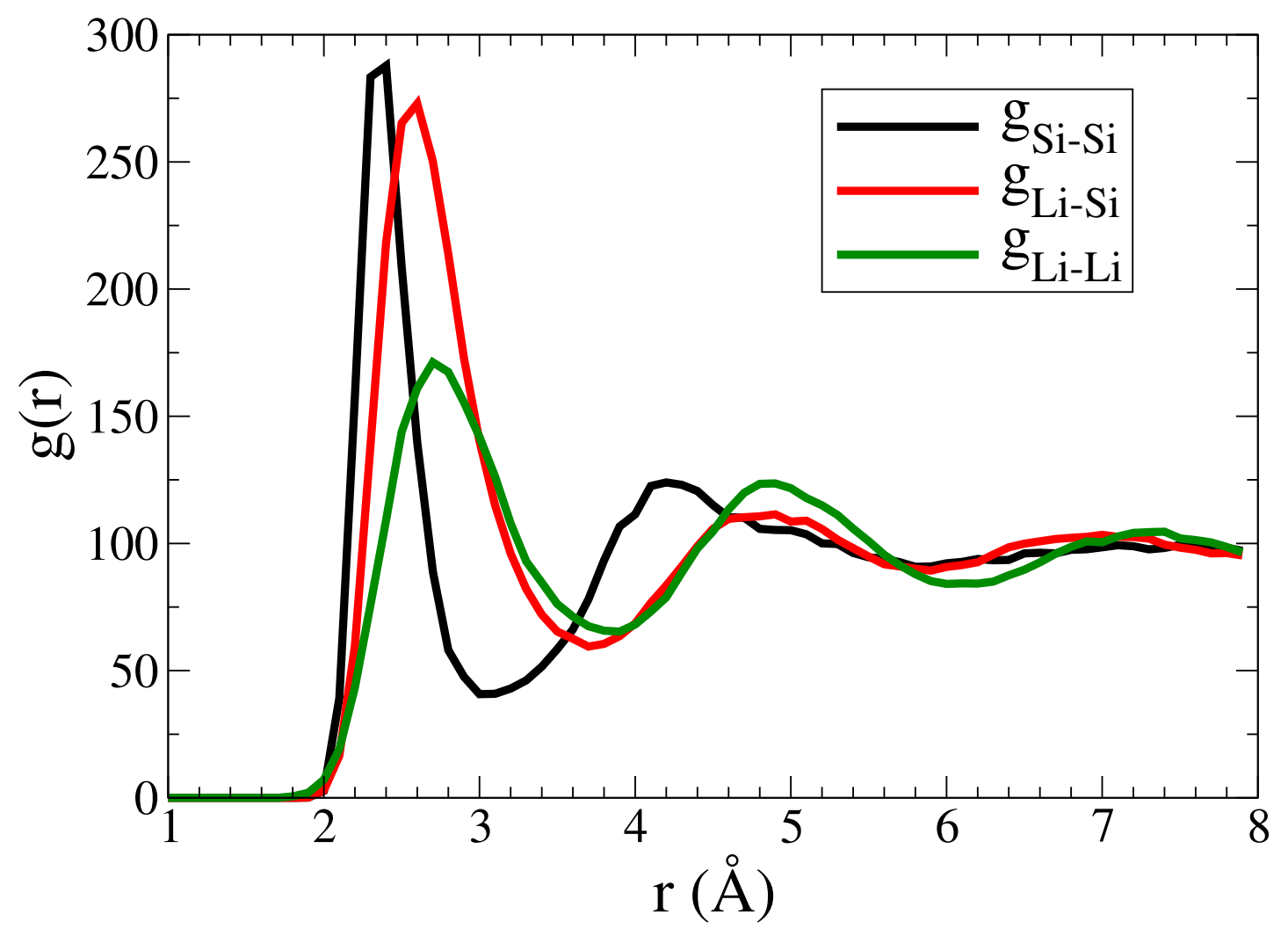

Figure 1: The Partial Radial Distribution Function of Si-Si, Li-Si, and Li-Li pairs in a-LiSi at $1200 \mathrm{~K}$ 


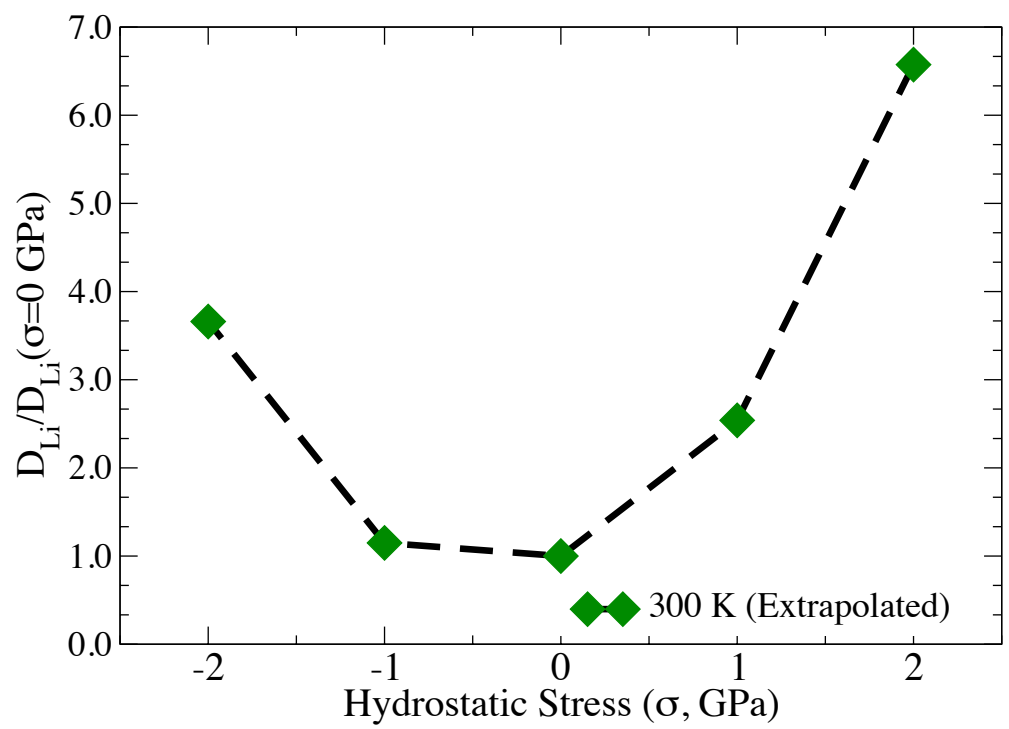

(a)

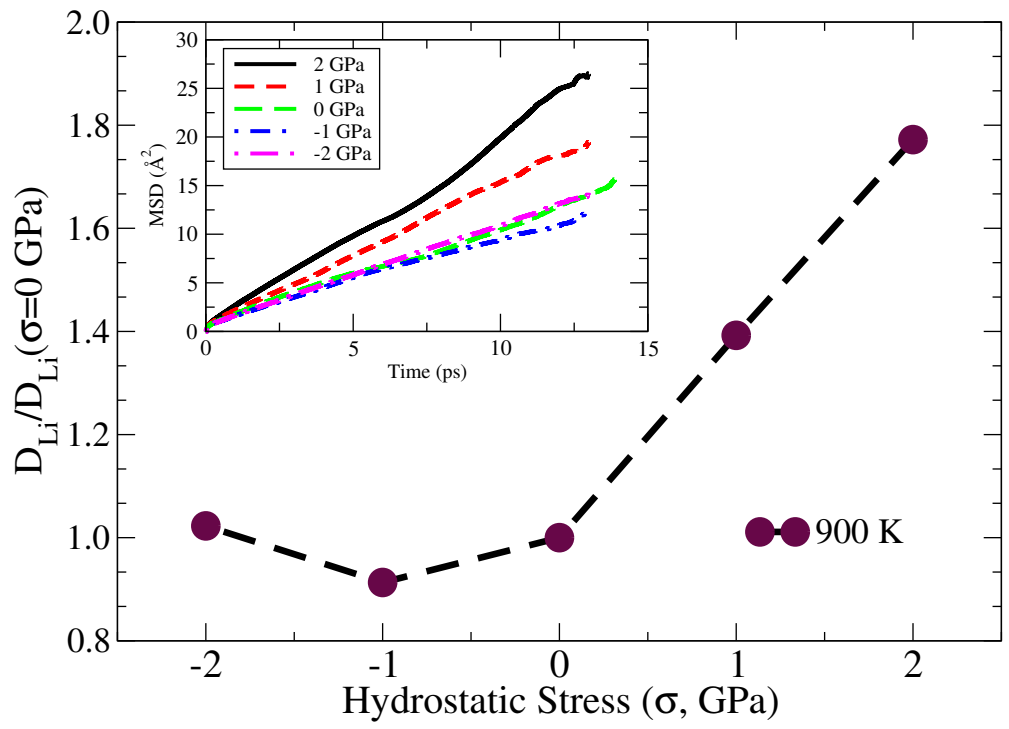

(b)

Figure 2: (a) The relative diffusion coefficients $\left(D_{L i} / D_{L i}(\sigma=0 G P a)\right)$ as a function of stress at $300 \mathrm{~K}$. (b) The relative diffusion coefficients $\left(D_{L i} / D_{L i}(\sigma=0 G P a)\right)$ as a function of stress at $900 \mathrm{~K}$. Insert: The MSD of $\mathrm{Li}$ in a-LiSi at $900 \mathrm{~K}$, the lowest temperature in our simulation, with different hydrostatic (positive value means tensile stress and negative value means compressive stress) stress states. 


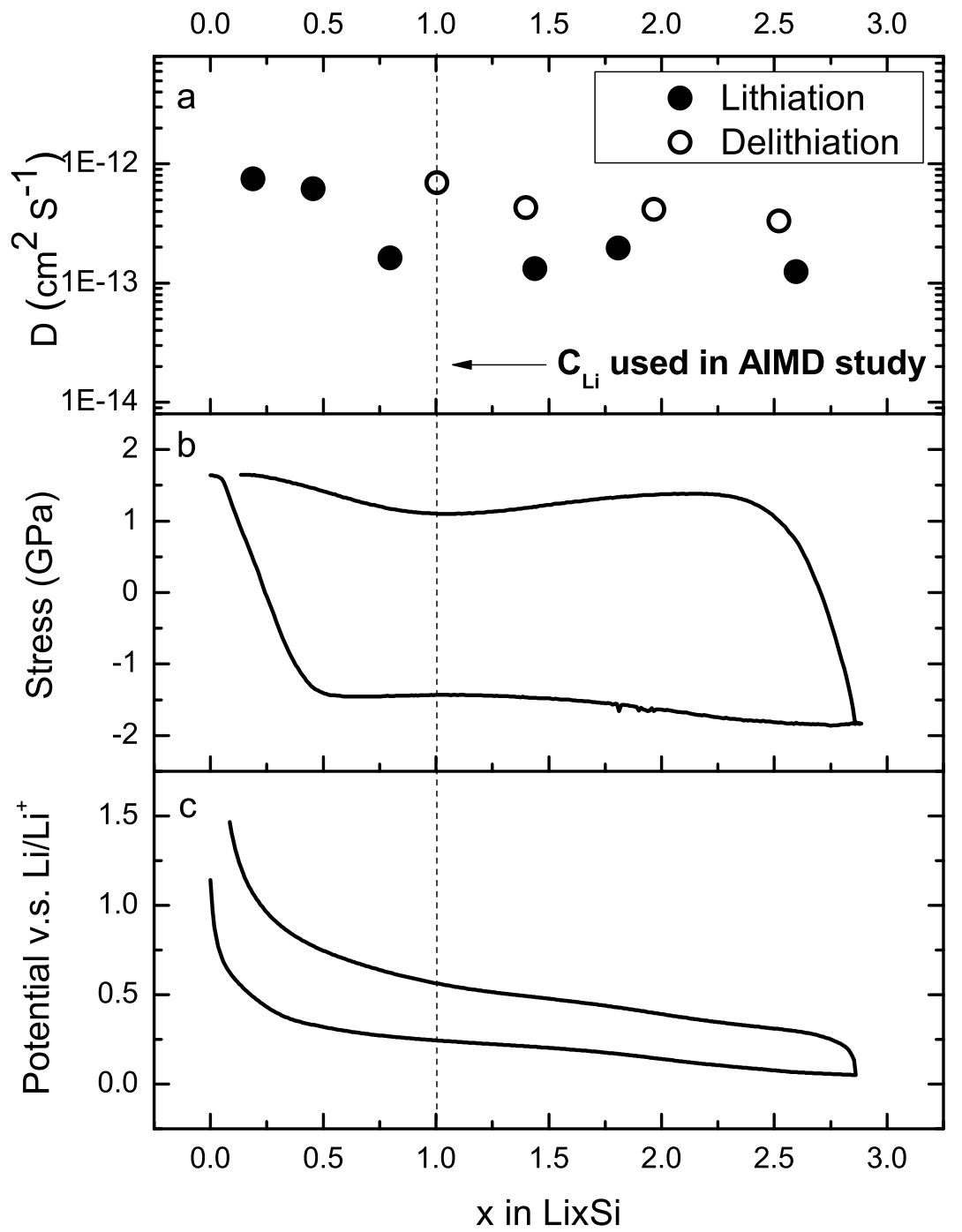

Figure 3: (a) Diffusion coefficients during lithiation and delithiation at different Li concentrations; (b) nominal stress calculated by Stony equation vs. $x$ in $\mathrm{Li}_{x} \mathrm{Si}$ under charge/discharge rate of $\mathrm{C} / 10$. (c) cell voltage vs. $x$ in $\mathrm{Li}_{x} \mathrm{Si}$ during stress measurement. 


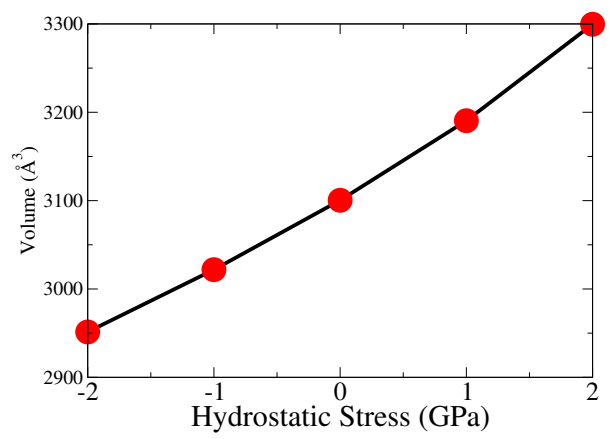

(a) Volumetric change due to stresses

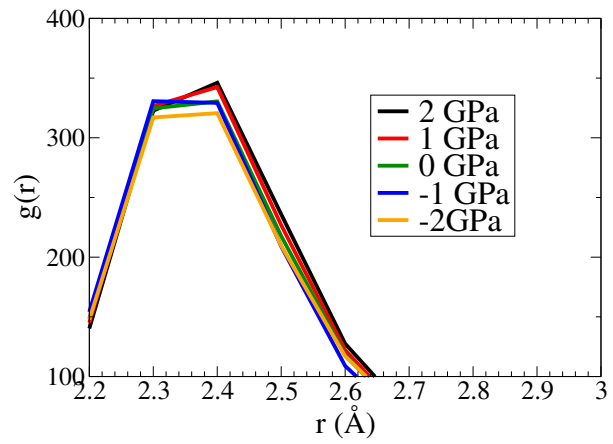

(b) Si-Si pair

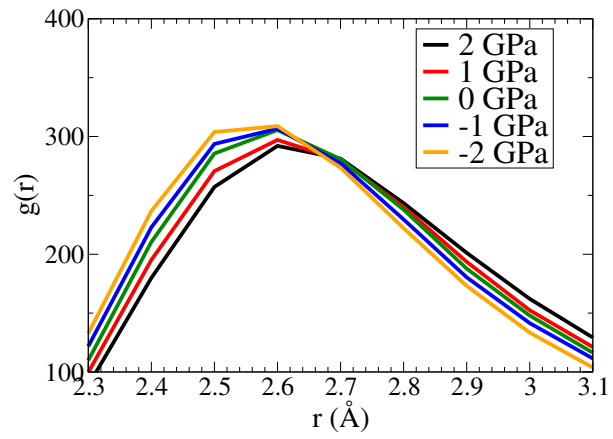

(c) Si-Li pair

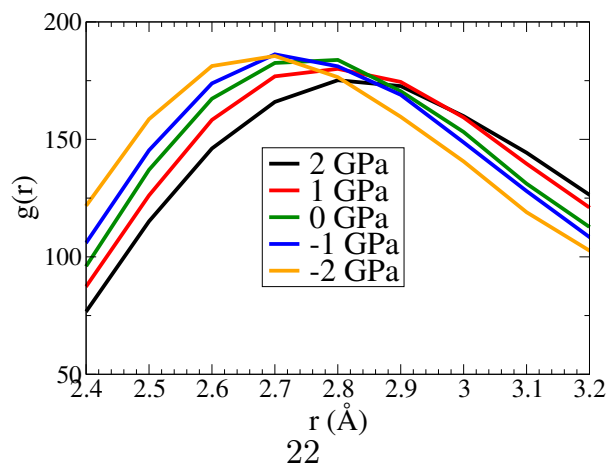

(d) Li-Li pair

Figure 4: (a) The volume of the simulation boxes under hydrostatic stress at $900 \mathrm{~K}$. (b)-(d) The change of the first peak in partial RDFs under different hydrostatic pressure states at $900 \mathrm{~K}$. 


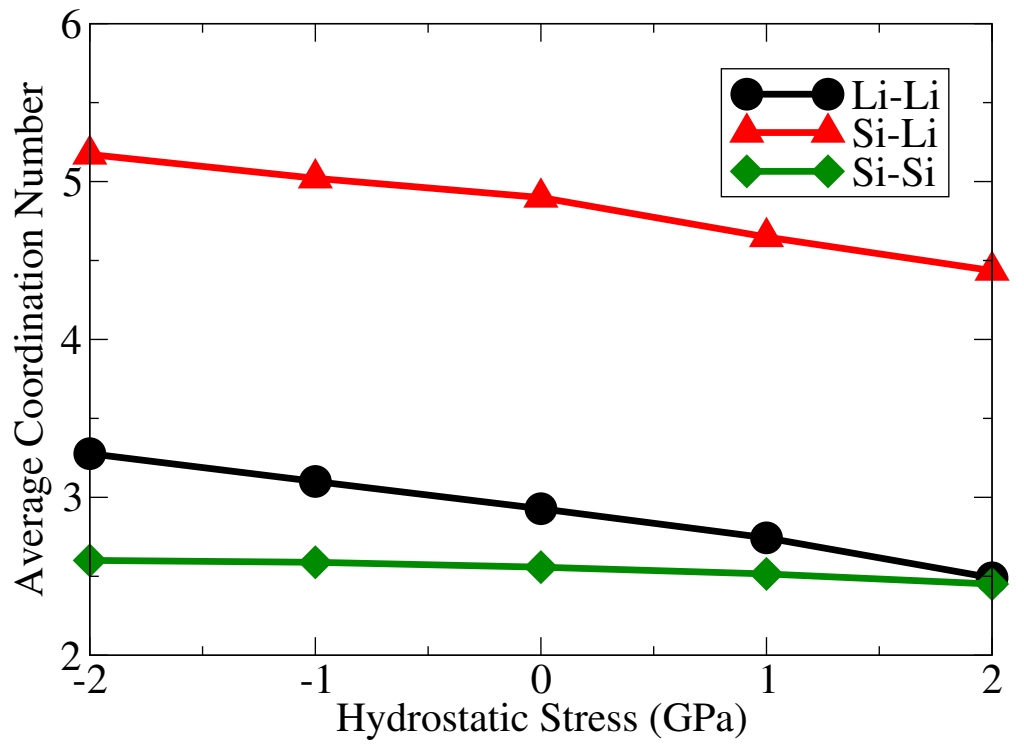

(a) Coordination number

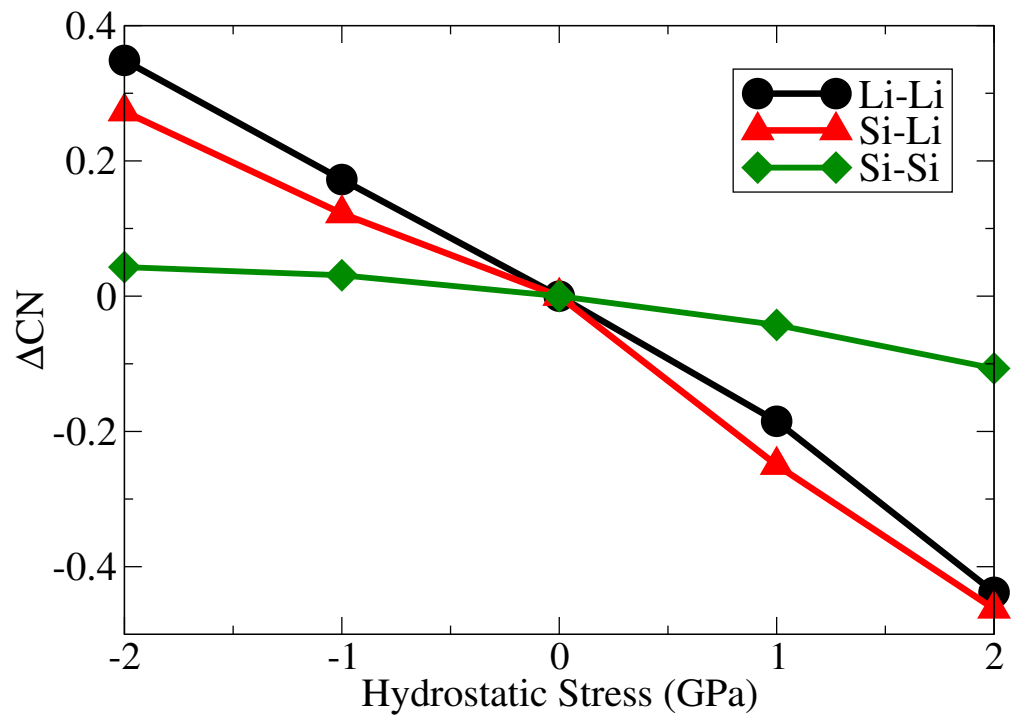

(b) $\triangle \mathrm{CN}$

Figure 5: (a) The coordination number as a function of stress at $900 \mathrm{~K}$; (b) $\Delta \mathrm{CN}$ with reference of $\mathrm{CN}$ at stress free state. 


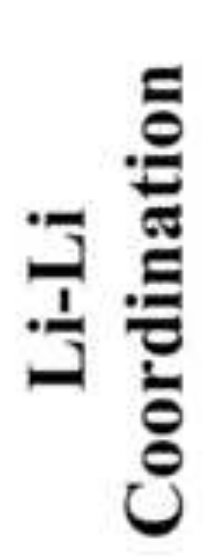

Lithiation
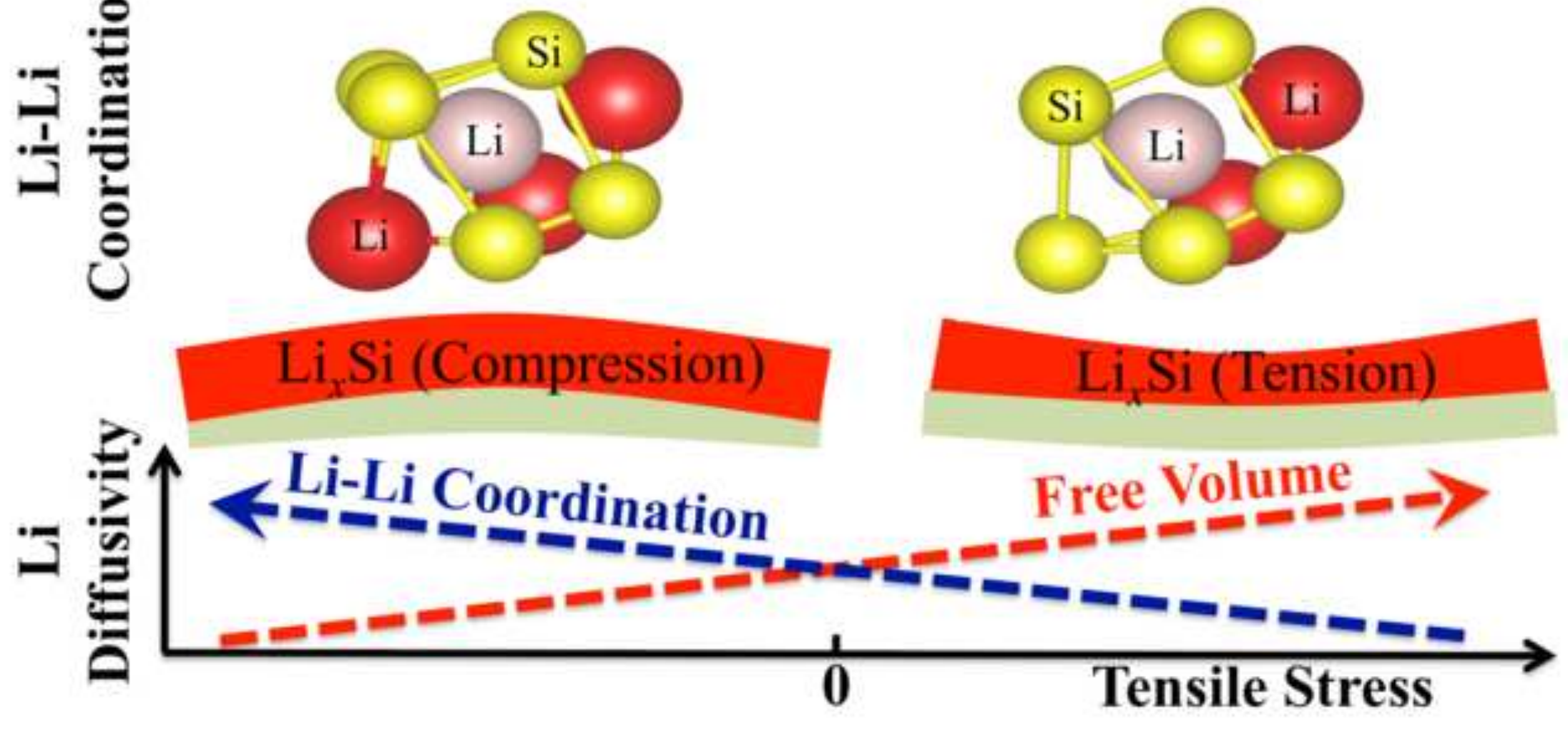